\title{
Perfil sociodemográfico y económico de estudiantes universitarios
}

\section{Socio-demographic and economic profile of university students}

\author{
Blanca Pérez-Contreras \\ $\triangle$ blanca.perez@cecar.edu.co \\ Corporación Universitaria del Caribe (CECAR), Sincelejo, Sucre, Colombia \\ Katia González-Otero (iD \\ $\triangle$ katia.gonzalez@cecar.edu.co \\ Corporación Universitaria del Caribe (CECAR), Sincelejo, Sucre, Colombia \\ Yaneth Polo-Bolaño \\ $\bowtie$ yanethpolo@unisinu.edu.co \\ Universidad del Sinú - Elías Bechara Zainúm, Montería, Córdoba, Colombia
}

Recibido: 08-02-2018

Aceptado: 28-05-2018

Publicado: 01-06-2018

\section{RESUMEN}

Objetivo. Este artículo es producto de la investigación que tuvo como objetivo identificar las características de la población estudiantil de pregrado y posgrado en la Corporación Universitaria del Caribe (CECAR), en Sincelejo (Sucre, Colombia), en el año 2016, mediante el análisis comparativo de variables demográficas, socio-familiares y económicas. Método. se utilizó un enfoque cuantitativo de corte positivista y tipo de estudio descriptivo; la muestra fue probabilística aleatoria simple de 1664 estudiantes, constituida por 851 de pregrado, modalidad presencial; 834 de la modalidad de educación a distancia, y 48 de especializaciones. El instrumento fue una encuesta construida con preguntas semiabiertas y cerradas, validado mediante juicio de expertos. Resultados. las características sociodemográficas no son significativamente diferenciales entre estudiantes de una y otra modalidad respecto a vínculos y dependencia del núcleo familiar, estado civil, procedencia y tipología familiar; en lo económico, prevalecen los estratos 1 y 2 , el ingreso económico no asciende a los dos salarios mínimos legales vigente, el nivel y la modalidad educativa tampoco son factores diferenciadores. Conclusiones. el estudio permitió mayor conocimiento sobre las características o perfil de los estudiantes, lo que sirvió a la vez para la trazabilidad de líneas de acción que favorezcan la atención de los más vulnerables social y económicamente, así como a la reorientación del apoyo académico, psicosocial, becas y auxilios.

Palabras clave: Características sociodemográficas y económicas; población estudiantil universitaria. 
Pérez-Contreras et al - Perfil sociodemográfico y económico de estudiantes universitarios

\section{ABSTRACT}

Objetive. This article is the product of the research that aimed to identify sociodemographic and economic characteristics of the student population of Corporación Universitaria del Caribe (CECAR), in Sincelejo (Sucre, Colombia), in 2016, comparatively analyzing them. Method. a quantitative positivist approach and type of descriptive study were used; the sample was simple random probabilistic of 1664 students constituted by 851 undergraduates, face-to-face modality; 834 of the distance education modality, and 48 of specializations. The instrument was a survey constructed with semi-open and closed questions, validated by expert judgment. Results. sociodemographic characteristics are not significantly different between students of one modality and another with respect to links and dependence of the family nucleus, marital status, origin and family typology; economically, stratum 1 and 2 prevail; the economic income does not amount to the two legal minimum wages in force; the level and the educational modality are not differentiating factors either. Conclusions. the study allowed greater knowledge about the characteristics or profile of the students, which served at the same time for the traceability of lines of action that favor the attention of the most vulnerable socially and economically, as well as reorient the academic, psychosocial support, scholarships and aid.

Keywords: Sociodemographic and economic characteristics, university student population

\section{INTRODUCCIÓN}

El nuevo perfil socioeconómico del estudiantado en América Latina y el Caribe, en el periodo que comprende la década de los ochenta hasta el inicio del siglo XXI, ha presentado transformaciones de gran importancia. La innovación demográfica que se vive actualmente y que caracterizan al nuevo estudiantado que ingresa a la educación superior en sus diferentes niveles y modalidades, implica, contar con información suficiente y organizada sobre los contextos de vida de la comunidad estudiantil que llega a las aulas y quienes, por razones diversas, en ocasiones, no se adaptan ni permanecen mucho tiempo en el sistema.

Las condiciones multiculturales, familiares, económicas y académicas del estudiantado, al ser cada vez más cambiantes y variables, exigen a las Instituciones de Educación Superior (IES) modificar sus formas tradicionales de abordar y dinamizar su función misional si quieren estar en equivalencia con el desarrollo global. En esta perspectiva, las universidades y el mismo Estado, tienen el reto de generar los cambios necesarios para responder sin restricciones a las nuevas demandas de una población estudiantil diversa y aumentar la capacidad de respuesta frente a sus necesidades y especificidades para garantizar igualdad y mejorar la calidad de la educación, lo que es consecuente con los Objetivos de Desarrollo Sostenible (Naciones Unidas, 2018, objetivo 4) y con los propósito del sistema educativo colombiano en cuanto a la propuesta de mayor acceso y mejor calidad del aprendizaje en las IES (Ley 30 de 1992).

La Asociación Colombiana de Universidades (ASCUN), año 2010-2014, dentro de su propuesta de política pública para la educación superior en Colombia, también se refiere a la necesidad de estructurar una nueva política de educación en este nivel que 
garantice "el acceso con equidad a una educación superior de calidad", lo que exige la caracterización y diferenciación de condiciones de ingreso y permanencia; la articulación con niveles precedentes, y la calidad y pertinencia de la oferta de cara a las necesidades del país y de las regiones. Atendiendo tales exigencias, la Corporación Universitaria del Caribe (CECAR) asume dentro de sus procesos internos y políticas la realización de investigaciones que posibiliten conocer ampliamente el perfil demográfico, social y económico de los estudiantes y su entorno familiar.

La descripción de perfil sociodemográfico, según Martínez, M. (2006), se centra en el análisis etimológico de la palabra, la cual se puede descomponer en dos términos: "socio" que quiere decir de la sociedad y "demografía" que significa estudio estadístico sobre un grupo de población humana. En consecuencia: "socio demográfico será un estudio estadístico de las características sociales de una población, es decir, cuántos tienen estudios medios, universitarios, cuántos trabajan, cuántos están desempleados, cuántos tienen casa propia y cuántos la tienen de alquiler/renta. Habrá tantas variantes como aspectos se quieran estudiar" (p. 258).

Lo socioeconómico, por su parte, se refiere a las características económicas o monetarias de la población estudiantil asociadas a sus condiciones sociales. Se afirma que el perfil socioeconómico: "es la identificación de los diferentes modos en que la sociedad ha organizado la producción y distribución de los bienes" (Willianson, 1991, p. 137). En este sentido, podemos citar el Informe sobre la Educación Superior en América Latina y el Caribe 2000-2005 de la UNESCO (2005) que define el perfil socioeconómico como "El conjunto de factores sociales (idiosincrasia de una población, cultura, modus vivendi, vestuario, educación, religión, lengua, educación, salud, seguridad, entre otros) de un país, [...], analizados como causa y a la vez consecuencia uno del otro" (p. 492).

Asimismo, un estudio realizado por estudiantes de cuarto semestre de la Facultad de Administración, denominado "perfil socioeconómico de mi comunidad", este, es definido como "atributo del hogar o de la familia que caracteriza su inserción social o económica y está basado en indicadores referidos al nivel de educación, el nivel de ocupación y el patrimonio" (Díaz, J. et al (2016). Afirma el autor que para su medición se deben tener en cuenta último año de estudio del jefe de familia, las características de la vivienda como, por ejemplo, número de cuartos, de baños, material de que está hecha, servicios, ingresos, entre otras variables (p.3).

De las anteriores conceptualizaciones, se colige que la condición social guarda una estrecha relación con la económica, y estas dos se encuentran, condicionadas por las características de su núcleo familiar. Así, el nivel formativo de los padres, la actividad económica a la que se dedican, los ingresos y bienes familiares se constituyen en oportunidades u obstáculos para el desarrollo personal y profesional de los hijos o, en efecto, para el futuro estudiante, que, en última instancia, es en quien recaen las consecuencias de un sistema educativo excluyente y con pocas opciones de permanencia en él. Sobre este asunto, hay un reconocimiento explícito respecto a que las políticas no son suficientes o no son formuladas a partir de dar respuestas a necesidades que se muestran homogéneas e indiferenciadas, en un país de naturaleza diversa y multicultural. Frente a esto, la propuesta de una nueva política pública para la educación superior debería prever a nivel institucional tales cambios e ir preparándose en la implementación de acciones internas que permitan crear las condiciones requeridas y actuar en coherencia 
Pérez-Contreras et al - Perfil sociodemográfico y económico de estudiantes universitarios

con los retos actuales que debe afrontar el sector educativo. Sobre este aspecto, un estudio sobre perfil socioeconómico del estudiante autónomo plantea que: "a nivel nacional suceden circunstancias y coyunturas particulares que imponen características adicionales a las demandas educativas y definen retos adicionales a las Instituciones de Educación Superior" (Asociación Colombiana de Universidades, 2007). Se percibe, por lo tanto, una deuda por cumplir en el contexto de Latinoamérica y en particular Colombia y el departamento de Sucre. Subsanarla, implica ajustar la educación a las necesidades del mundo cambiante, pero, además, a las características sociales, culturales, biográficas y económicas de los estudiantes del siglo xxI.

En esta perspectiva, un aspecto a considerar es la inclusión de investigaciones de este tipo en los planes de desarrollo institucionales y en los planes estratégicos de ciencia, tecnología e innovación a fin de tener conocimiento de las necesidades y condiciones de vida de los estudiantes en y desde su entorno familiar. En este marco, existen evidencias empíricas de algunos estudios realizados por universidades a nivel nacional, que describen aspectos relevantes de los estudiantes universitarios. Uno de ellos es la investigación, "Características del perfil socioeconómico del estudiante de pregrado, realizada por la Oficina de Planeación de la Universidad de Pamplona, (2012), el cual agrupa características demográficas, académicas, socioeconómica, de bienestar y comportamiento universitario de la población estudiantil (p. 61).

Por su parte, Rahona (2006) en su estudio "Efectos del entorno socioeconómico en la realización de estudios universitarios" agrupa elementos en tres categorías: a) determinantes familiares, con énfasis en las ganancias familiares, el grado de estudios y el estatus socioeconómico de los jefes de hogar; b) determinantes personales, en los que se contrasta empíricamente la importancia del género en la realización de estudios superiores $y$, por último, c) determinantes relacionados con el entorno del estudiante en los que se destaca el año en que el estudiante concluyó su educación media, la elección del estudio según interés y las condiciones institucionales.

Otras investigaciones como la realizada por Boucourt y González (2006) tocan características de la población estudiantil al hacer referencia a variables demográficas, socioeconómicas, culturales, académicas, psicológicas, salud, uso del tiempo libre, condiciones de vida, antecedentes escolares, actitud hacia la profesión y la universidad. De igual manera, instituciones como la Universidad Nacional de Colombia (2001), la Universidad del Magdalena (2004), la Escuela de Administración, Finanzas y Tecnología (EAFIT) (2007) y la Universidad Autónoma de Occidente (2007) han realizado análisis socioeconómicos acerca de su comunidad estudiantil dando origen a una riqueza de información útil a nivel institucional que ha sido columna para la ejecución de otras investigaciones en el ámbito colombiano.

En este espíritu de búsqueda de nuevos conocimientos sobre el estudiantado universitario, se ancla la investigación realizada en CECAR en el año 2016, que trata de "las características socioeconómicas de los estudiantes y su núcleo familiar" y tuvo como objetivo explorar y describir variables demográficas, sociales, familiares y económicas, mediante el uso de la estadística muestral para construir el perfil socioeconómico del estudiante de dicha universidad, que contribuya a definir política de bienestar universitario. 


\section{MÉTODO}

La caracterización del perfil socioeconómico del estudiante de CECAR se trabajó con enfoque cuantitativo, en un tipo de estudio descriptivo. A través de él, se busca medir con la mayor precisión posible las características de un fenómeno con el fin de decir cómo es y cómo se manifiesta (Hernández, Fernández y Baptista, 1999, p. 61). En nuestro caso, interesan las características demográficas, sociales y económicas del estudiante de esta universidad en y desde su entorno familiar.

Población y muestra. Durante el primer semestre académico de 2016, la población estudiantil de CECAR se estimó en 11.616 estudiantes, incluidos el nivel de pregrado modalidad presencial, a distancia, virtual, educación continuada y posgrado. No obstante, la investigación tomó como referente a 6.584 estudiantes de pregrado modalidad presencial, educación a distancia y especializaciones. Para la definición del tamaño de la muestra se utilizó muestreo probabilístico aleatorio simple con aplicación de la fórmula estadística:

$\mathrm{n}=\left(\mathrm{N} * \mathrm{Z}^{2} * \mathrm{P} * \mathrm{q}\right) /\left(\mathrm{Z}^{2} * \mathrm{p} * \mathrm{q}\right)+\left(\mathrm{e}^{2}(\mathrm{~N}-1)\right)$ Donde;

$\mathrm{n}=$ Tamaño de la muestra a calcular

$Z=$ valor asignado para un nivel de confianza del $95 \%$ para la población, donde $Z=1,96$

$\mathrm{N}=$ tamaño de la población, donde $\mathrm{N}=6.584$

$p=$ probabilidad de que un estudiante sea seleccionado, donde $p=0,5$

$q=$ probabilidad de que un estudiante no sea seleccionado, donde $q=1$ y $p=0,5$

$\mathrm{e}=$ error asociado a la muestra, donde $\mathrm{e}=0,05$

Al reemplazar los valores respectivos en la fórmula de determinación del tamaño de la muestra, se observa que esta es de 1733 estudiantes, distribuidos así: 851 de modalidad presencial, 834 de educación abierta y a distancia, y 48 de especializaciones. Los tablas 1,2 y 3 registran el número total de estudiantes por programa y la muestra seleccionada por programa, modalidad y nivel.

Tabla 1. Distribución de la muestra en pregrado presencial.

\begin{tabular}{|c|c|c|c|c|c|c|c|c|c|c|c|c|}
\hline \multicolumn{13}{|c|}{ Definición del número de unidades de análisis por muestreo estratificado } \\
\hline \multirow{2}{*}{ Programas/ Presencial } & \multicolumn{11}{|c|}{ Semestre } & \multirow{2}{*}{$\mathbf{N}$} \\
\hline & 1 & 2 & 3 & 4 & 5 & 6 & 7 & 8 & 9 & 10 & 11 & \\
\hline Administración de empresas & 3 & 3 & 2 & 2 & 1 & 1 & 1 & 3 & 2 & & & 18 \\
\hline Arquitectura & 4 & 18 & 3 & 5 & 3 & 7 & 2 & 3 & 2 & & & 47 \\
\hline Ciencias del Deporte y la Actividad Física & 4 & 4 & 3 & 4 & 1 & 2 & 1 & 2 & 1 & & & 22 \\
\hline Contaduría Pública & 7 & 11 & 5 & 15 & 5 & 14 & 13 & 16 & 17 & & & 103 \\
\hline Derecho & 26 & 72 & 26 & 61 & 15 & 44 & 39 & 77 & 44 & & & 404 \\
\hline Economía & 1 & 1 & & & & & & & & & & 2 \\
\hline Ingeniería de Sistemas & 1 & 3 & & 1 & & 1 & & 1 & & & & 7 \\
\hline Ingeniería Industrial & 3 & 10 & 4 & 12 & 2 & 5 & 3 & 4 & 3 & & & 46 \\
\hline Lic. Básica: Educación Artística & & & & 1 & 1 & & & & & & & 2 \\
\hline Lic. Básica: Humanidades y Lengua Castellana e Inglés & & & & & 1 & & 2 & & 1 & & & 4 \\
\hline Licenciatura en Lingüística y Literatura & & 1 & & & & & & & & & & 1 \\
\hline Licenciatura en Pedagogía Infantil & 1 & & & & & & & & & & & 1 \\
\hline Psicología & 9 & 32 & 12 & 17 & 11 & 15 & 11 & 14 & 10 & & & 131 \\
\hline Trabajo Social & 6 & 12 & 6 & 16 & 3 & 9 & 5 & 3 & 3 & & & 63 \\
\hline Total por semestre & 62 & 167 & 58 & 133 & 43 & 95 & 73 & 123 & 76 & 0 & $\mathbf{0}$ & 851 \\
\hline
\end{tabular}

Fuente: cálculo estadístico, grupo de investigación 
Pérez-Contreras et al - Perfil sociodemográfico y económico de estudiantes universitarios

Tabla 2. Distribución de la muestra en educación a distancia.

\begin{tabular}{|c|c|c|c|c|c|c|c|c|c|c|c|c|}
\hline \multicolumn{13}{|c|}{ Definición del número de unidades de análisis por muestreo estratificado } \\
\hline \multirow{2}{*}{ Programas/ Distancia } & \multicolumn{11}{|c|}{ Semestre } & \multirow{2}{*}{ n } \\
\hline & 1 & 2 & 3 & 4 & 5 & 6 & 7 & 8 & 9 & 10 & 11 & \\
\hline Administración de Empresas & 62 & 1 & 41 & 2 & 17 & 27 & 1 & 8 & 1 & 0 & 0 & 160 \\
\hline Administración Pública & 5 & 3 & & & 2 & & & & 5 & 0 & 0 & 15 \\
\hline Ciencias Naturales y Educación Ambiental & & 7 & 2 & 25 & 12 & 13 & 9 & 26 & 32 & 0 & 0 & 126 \\
\hline Tecnología e Informática & & & 1 & 2 & 1 & 2 & 4 & 12 & 4 & 0 & 0 & 26 \\
\hline Humanidades, Lengua Castellana e Inglés & & 10 & 8 & 17 & 39 & 41 & 100 & 170 & 91 & 0 & 0 & 476 \\
\hline Total por semestre & 67 & 21 & 52 & 47 & 78 & 88 & 125 & 219 & 137 & 0 & 0 & 834 \\
\hline
\end{tabular}

Tabla 3. Distribución de la muestra en especializaciones

\begin{tabular}{lcc}
\hline \multicolumn{1}{c}{ ESPECIALIZACIÓN } & POBLACIÓN & MUESTRA \\
\hline Especialización en Derecho Procesal Civil & 35 & 7 \\
Especialización en Administración Financiera & 28 & 5 \\
Especialización en Gerencia de la Calidad y Auditoría en Salud & 60 & 2 \\
Especialización en Investigación Aplicada a la Educación & 13 & 1 \\
Especialización en Derecho Laboral y Seguridad Social & 3 & 1 \\
Especialización en Procesos Familiares y Comunitarios & 3 & 4 \\
Especialización en Gerencia Ambiental & 25 & 9 \\
Especialización en Derecho Administrativo & 40 & 1 \\
Especialización de la Hacienda Pública & 4 & 5 \\
Especialización en Gerencia Pública & 32 & 3 \\
Especialización en Docencia & 25 & 48 \\
\hline Total & 378 & \\
\end{tabular}

Fuente: cálculo estadístico, grupo de investigación

Instrumentos. La información se recolectó mediante un instrumento de encuesta constituido por preguntas semiabiertas y cerradas, que se dispuso en la página web de CECAR, para ser respondido por aquellos estudiantes matriculados en los centros de atención más lejanos; otros fueron aplicados directamente en el campus universitario y en visita domiciliaria. Esta información se complementó con la base de datos del sistema de información académica, elaborada por CECAR. El instrumento fue validado previamente mediante un pilotaje realizado con un grupo de diez estudiantes, con el fin de verificar la comprensión en el uso del lenguaje, la claridad y pertinencia de las preguntas.

Atendiendo la descripción del perfil sociodemográfico y socioeconómico que hacen los autores citados, la investigación realizada incluye cuatro dimensiones: la demográfica, social, familiar y económica, que al operacionalizarse se construyeron como los indicadores e ítems. 
Procedimiento. Se seleccionó la muestra por programa a partir de la base de datos suministrada por CECAR y en correspondencia con el número total de estudiantes por programas. Se recolectó la información mediante una encuesta semiestructurada, la que se organizó, codificó y vació en una planilla de cálculo Excel XP, diseñada de acuerdo a las preguntas contenidas en el instrumento y los objetivos de la investigación. Hecho esto, se procedió a la elaboración de tablas y gráficas en las que se sintetizan las características del estudiante y su núcleo familiar. Posteriormente, se hizo el análisis comparado entre pregrado presencial y a distancia y de estos con posgrado, con el fin de identificar acercamientos o diferencias entre los estudiantes de unos y otros programas, según modalidad y nivel de formación. A partir de los resultados emergieron las líneas de acción que se re recomiendan a los programas e institución.

\section{RESULTADOS}

Los resultados muestran el análisis comparado para los tres grupos de población estudiantil participantes en la investigación, atendiendo características demográficas, sociales, familiares y económicas. En lo familiar se hace referencia a aspectos educativos y laborales de los padres, ingresos percibidos y apoyo económico al estudiante.

Características demográficas. Comparando la variable género, se observa una participación mayor de mujeres tanto en pregrado como en posgrado, con una representación total de 1.093 , es decir que entre el $74 \%$ y el $55,2 \%$ son mujeres, frente a un porcentaje de hombres menor (ver Tabla 4).

Tabla 4. Comparativo de los estudiantes según género, CECAR 2016-I.

\begin{tabular}{ccccc}
\hline & \multicolumn{3}{c}{ Género } \\
\cline { 2 - 5 } Modalidad & \multicolumn{2}{c}{ MASCULINO } & \multicolumn{3}{c}{ FEMENINO } \\
\cline { 2 - 5 } & Frecuencia absoluta & $\%$ & Frecuencia absoluta & $\%$ \\
\hline PRESENCIAL & 381 & $44,8 \%$ & 470 & $55,2 \%$ \\
A DISTANCIA & 209 & $26,0 \%$ & 594 & $74,0 \%$ \\
POSGRADO & 16 & $35,6 \%$ & 29 & $64,4 \%$ \\
\hline
\end{tabular}

Fuente: cálculo de las autoras con base en la encuesta

En cuanto a la edad, pregrado a distancia tiene el número más alto de personas entre los veintitrés y los 36 años, con una representación de $60 \%$; en presencial la mayoría son jóvenes, sus edades oscilan entre dieciséis y veintidós años $(66,5 \%)$; la población estudiantil mayor de 37 años está más concentrada en posgrado y a distancia. Un cambio observado en el perfil demográfico de estudiantes de pregrado es la tendencia de ingreso de personas mayores de treinta años a esta modalidad (ver Tabla 5). 
Tabla 5. Edad de los estudiantes por modalidad y nivel, CECAR 2016-I

\begin{tabular}{|c|c|c|c|c|c|c|c|c|c|c|}
\hline \multirow{3}{*}{ Modalidad } & \multicolumn{10}{|c|}{ Edad } \\
\hline & \multicolumn{2}{|c|}{16 a 22 años } & \multicolumn{2}{|c|}{23 a 29 años } & \multicolumn{2}{|c|}{30 a 36 años } & \multicolumn{2}{|c|}{37 a 43 años } & \multicolumn{2}{|c|}{$>43$ años } \\
\hline & No. & $\%$ & No. & $\%$ & No. & $\%$ & No. & $\%$ & No. & $\%$ \\
\hline PRESENCIAL & 566 & 66,5 & 180 & 21,2 & 59 & 6,9 & 22 & 2,6 & 24 & 2,8 \\
\hline A DISTANCIA & 249 & 31 & 361 & 45,0 & 121 & 15,1 & 47 & 5,9 & 25 & 3,1 \\
\hline POSGRADO & 6 & 13,3 & 23 & 51,1 & 5 & 11,1 & 4 & 8,9 & 7 & 15,6 \\
\hline
\end{tabular}

Según los datos registrados en la Tabla 6, el comportamiento del estado civil en estudiantes de pregrado y posgrado muestra mayor presencia de población soltera en los tres grupos analizados, mientras que el número de casados y en unión libre es mayor en educación a distancia.

Tabla 6. Estado civil de los estudiantes por modalidad y nivel, CECAR 2016-I

\begin{tabular}{|c|c|c|c|c|c|c|c|c|c|c|}
\hline \multirow{3}{*}{ Modalidad } & \multicolumn{10}{|c|}{ Estado civil } \\
\hline & \multicolumn{2}{|c|}{ Soltero } & \multicolumn{2}{|c|}{ Casado } & \multicolumn{2}{|c|}{ Unión libre } & \multicolumn{2}{|c|}{ Separado } & \multicolumn{2}{|c|}{ Viudo } \\
\hline & No. & $\%$ & No. & $\%$ & No. & $\%$ & No. & $\%$ & No. & $\%$ \\
\hline Presencial & 705 & 82,8 & 66 & 7,8 & 69 & 8,1 & 11 & 1,3 & 0 & 0 \\
\hline Distancia & 483 & 60,1 & 140 & 17 & 162 & 20 & 17 & 2,1 & 1 & 0 \\
\hline Posgrado & 26 & 57,8 & 14 & 31 & 4 & 8,9 & 1 & 2,2 & 0 & 0 \\
\hline
\end{tabular}

Fuente: cálculo de las autoras con base en la encuesta.

Respecto a las características demográficas, en su Informe sobre la Educación Superior en América Latina y el Caribe (2000-2005), Rama (2006) afirma que "el incremento de la matricula ha sido muy superior al incremento de la población de 20 a 24 años" y que el proceso de masificación de la educación superior: "Se manifiesta en cambios en el perfil social del estudiantado: estudiantes de corto tiempo, estudiantes trabajadores, estudiantes a distancia, estudiantes indígenas, diversidad racial, estudiantes con discapacidades, junto a los tradicionales estudiantes blancos, urbanos y de familias de altos ingresos" (p. 15). Con relación a estas características, se puede concluir que a CECAR Ilega población de edades variadas, pero, además, en situación de discapacidad, adulta joven y mayor, además de los estudiantes tradicionales que anteriormente caracterizaban la conformación del grupo de aula.

Respecto a la variable "estado civil", otros estudios a nivel nacional identifican predominio del estado civil soltero frente a otros como casados, unión libre, divorciado, viudo. Este es el caso de la universidad de Pamplona y EAFIT, en las que el $92,53 \%$ el $96.8 \%$ son estudiantes solteros, respectivamente. Como se puede observar, estos porcentajes son mayores a los de CECAR. 
Características de familiares. En esta categoría se agrupan tipología familiar, aspectos educativos y laborales de los padres, ingresos percibidos y apoyo económico al estudiante.

Respecto a la primera se encontró que la población que estudia en CECAR, a pesar de ser diversificada y provenir de diferentes contextos socioculturales, presenta cierta homogeneidad en la tipología familiar. Predomina en los tres grupos poblacionales la nuclear, que supera el $73,3 \%$ en posgrado, el $82 \%$ en la modalidad a distancia y el $75 \%$ en presencial (obsérvese el registro de la Tabla 7). Las familias reconstituidas y extensas representan un mínimo porcentaje, sin embargo, el número de integrantes, el tipo de ocupación laboral e ingresos puede constituirse en un obstáculo en la satisfacción de necesidades básicas, incluida entre ellas la educativa.

Tabla 7. Tipología familiar de la población estudiantil, CECAR 2016-1

\begin{tabular}{|c|c|c|c|c|c|c|c|c|c|c|c|c|}
\hline \multirow{3}{*}{ Modalidad } & \multicolumn{12}{|c|}{ Tipo de familia } \\
\hline & \multicolumn{2}{|c|}{ Nuclear } & \multicolumn{2}{|c|}{ Extensa } & \multicolumn{2}{|c|}{ Reconstruida } & \multicolumn{2}{|c|}{ Monogámica } & \multicolumn{2}{|c|}{ Otra } & \multicolumn{2}{|c|}{ No responde } \\
\hline & No. & $\%$ & No. & $\%$ & No. & $\%$ & No. & $\%$ & No. & $\%$ & No. & $\%$ \\
\hline Presencial & 646 & 75,9 & 102 & 12,0 & 27 & 3,2 & 45 & 5,3 & 20 & 2,4 & 11 & 1,3 \\
\hline Distancia & 659 & 82,1 & 85 & 10,6 & 27 & 3,4 & 17 & 2,1 & 8 & 1,0 & 7 & 0,9 \\
\hline Posgrado & 33 & 73,3 & 7 & 15,6 & 1 & 2,2 & 4 & 8,9 & 0 & 0,0 & 0 & 0,0 \\
\hline
\end{tabular}

Fuente: cálculo de las autoras con base en la encuesta

Número de hijos en el núcleo familiar. En cuanto al número de hijos por familia, la Tabla 8 registra para pregrado y posgrado, por una parte, familias sin hijos y, por la otra, un número que oscila entre uno, dos y tres hijos, indistintamente de si viven en áreas urbanas o rurales o del grupo generacional al que pertenezca. Esta composición es la característica principal que define la familiar nuclear simple en Colombia, Gutiérrez de Pineda (1999, p. 381).

Tabla 8. Número de hijos de la población estudiantil, CECAR 2016-1

\begin{tabular}{|c|c|c|c|c|c|c|c|c|c|c|c|c|c|c|}
\hline \multirow{2}{*}{ Modalidad } & \multicolumn{2}{|c|}{0} & \multicolumn{2}{|c|}{1} & \multicolumn{2}{|c|}{2} & \multicolumn{2}{|c|}{3} & \multicolumn{2}{|c|}{4} & \multicolumn{2}{|c|}{5} & \multicolumn{2}{|c|}{6} \\
\hline & No. & $\%$ & No. & $\%$ & No. & $\%$ & No. & $\%$ & No. & $\%$ & No. & $\%$ & No. & $\%$ \\
\hline Presencial & 697 & 81,9 & 89 & 10,5 & 45 & 5,3 & 15 & 1,8 & 3 & 0,4 & 1 & 0,1 & 1 & 0,1 \\
\hline A distancia & 471 & 58,7 & 191 & 23,8 & 101 & 12,6 & 32 & 4,0 & 6 & 0,7 & 2 & 0,2 & 0 & 0,0 \\
\hline Posgrado & 21 & 46,7 & 10 & 22,2 & 8 & 17,8 & 6 & 13,3 & 0 & 0,0 & 0 & 0,0 & 0 & 0,0 \\
\hline
\end{tabular}

Fuente: cálculo de las autoras con base en la encuesta.

Respecto a la variable "hermanos que estudian en el núcleo familiar", se encontró que, entre uno, dos y más integrantes del núcleo familiar están matriculados en esta u otras instituciones educativas. Esta información debe ser verificada por la institución, con el fin de abrir rutas de atención para esta población estudiantil y aplicar algunos incentivos de los establecidos institucionalmente. Además, se conoció que un porcentaje relativamente bajo (entre $23 \%$ y $29,8 \%$ en pregrado y $24,4 \%$ en posgrado) no tiene hermanos estudiando a nivel de pregrado ni en primaria o secundaria, lo que puede ser factor de permanencia y continuidad de los estudios (Tabla 9). 
Tabla 9. Hermanos que estudian, CECAR 2016-1

\begin{tabular}{|c|c|c|c|c|c|c|c|c|c|c|c|c|c|c|c|c|c|c|c|c|}
\hline \multirow{2}{*}{ Modalidad } & \multicolumn{2}{|c|}{$\mathbf{0}$} & \multicolumn{2}{|c|}{1} & \multicolumn{2}{|c|}{2} & \multicolumn{2}{|c|}{3} & \multicolumn{2}{|c|}{4} & \multicolumn{2}{|c|}{5} & \multicolumn{2}{|c|}{6} & \multicolumn{2}{|c|}{7} & \multicolumn{2}{|c|}{8} & \multicolumn{2}{|c|}{9} \\
\hline & No. & $\%$ & No. & $\%$ & No. & $\%$ & No. & $\%$ & No. & $\%$ & No. & $\%$ & No. & $\%$ & No. & $\%$ & No. & $\%$ & No. & $\%$ \\
\hline Presencial & 189 & 23 & 257 & 31,3 & 222 & 27,1 & 101 & 12,3 & 29 & 3,5 & 16 & 2,0 & 4 & 0,5 & 2 & 0,2 & 0 & 0,0 & 0 & 0,0 \\
\hline Distancia & 231 & 29,8 & 191 & 24,6 & 246 & 31,7 & 58 & 7,5 & 28 & 3,6 & 10 & 1,3 & 8 & 1,0 & 1 & 0,1 & 1 & 0,1 & 1 & 0,1 \\
\hline Posgrado & 11 & 24,4 & 10 & 22,2 & 8 & 17,8 & 13 & 28,9 & 3 & 6,7 & 0 & 0,0 & 0 & 0,0 & 0 & 0,0 & 0 & 0,0 & 0 & 0,0 \\
\hline
\end{tabular}

Fuente: cálculo de las autoras con base en la encuesta

En términos generales, los resultados sobre características del entorno familiar de nuestros estudiantes y los de otras universidades como la EAFIT son similares, indistintamente del programa académico, la modalidad (presencial o a distancia) y el nivel (pregrado o posgrado). Los estudios consultados muestran que la mayoría de los estudiantes viven actualmente con su familia paterna y una tendencia a constituir familias nucleares siendo común con lo encontrado en CECAR. El estudio realizado por Acevedo y Jaramillo (2007), en la universidad EAFIT, afirma que la mayoría de los estudiantes $(74,5 \%)$ viven actualmente con su familia paterna, formada esta por padre, madre y hermanos. Un $11,9 \%$ corresponde a los estudiantes que residen con otros familiares como hermanos, primos y tíos; este último aspecto también es común en nuestros estudiantes. El estudio de la Universidad de Pamplona, por el contrario, indica, que el $48,44 \%$ vive con sus padres o hermanos, es decir que el porcentaje es menor al de CECAR y la EAFIT.

Características socioeconómicas. Al analizar la condición del estudiante por estrato, se encontró que la mayoría pertenecen al uno y dos, constituyéndose en un denominador común en los programas de pregrado presencial, a distancia y especializaciones; el número de estudiantes que pertenece al estrato tres en los programas estudiados no supera el $18 \%$, en tanto que son pocos los que pertenecen a estratos más elevados. Se infiere que CECAR acoge en sus aulas población en alta vulnerabilidad social y económica (Tabla 10).

Tabla 10. Estrato de los estudiantes, CECAR 2016-1

\begin{tabular}{cccccccccccccc}
\hline \multirow{2}{*}{ Modalidad } & \multicolumn{2}{c}{$\mathbf{1}$} & \multicolumn{2}{c}{$\mathbf{2}$} & \multicolumn{2}{c}{$\mathbf{3}$} & \multicolumn{4}{c}{$\mathbf{4}$} & \multicolumn{5}{c}{$\mathbf{5}$} \\
\cline { 2 - 13 } & No. & \% & No. & \% & No. & \% & No. & \% & No. & \% & No. & \% \\
\hline Presencial & 369 & 43,4 & 346 & 40,7 & 110 & 12,9 & 20 & 2,4 & 4 & 0,5 & 2 & 0,2 \\
Distancia & 388 & 48,3 & 277 & 34,5 & 121 & 15,1 & 14 & 1,7 & 3 & 0,4 & 0 & 0 \\
Posgrado & 11 & 24,4 & 21 & 46,7 & 8 & 17,8 & 3 & 6,7 & 2 & 4,4 & 0 & 0 \\
\hline
\end{tabular}

Fuente: cálculo de las autoras con base en la encuesta

Ocupación o vínculo laboral. Un total de $32 \%$ de estudiantes de pregrado presencial trabaja, mientras que en educación a distancia y posgrado más del $60 \%$ lo hace, es decir que se duplica el porcentaje. Este comportamiento explica los resultados obtenidos en cuanto a dependencia económica de los padres o familiares (ver tabla 11). 
Tabla 11. Porcentaje de estudiantes según vínculo laboral, CECAR 2016-1

\begin{tabular}{cccccc}
\hline & \multicolumn{4}{c}{ ¿Trabaja actualmente? } \\
\cline { 2 - 5 } Modalidad & No. & SI & \% & No. & \% \\
\cline { 2 - 5 } & 272 & 32 & 579 & 68 \\
Presencial & 529 & 65,9 & 274 & 34,1 \\
Distancia & 29 & 64,4 & 16 & 35,6 \\
Posgrado & & & & \\
\hline
\end{tabular}

Fuente: cálculo de las autoras con base en la encuesta

Dependencia económica del estudiante. Respecto a la variable dependencia económica, se evidencia en la tabla 12, una mayor dependencia de ambos padres en pregrado presencial, seguido de solo el padre o la madre. En la modalidad a distancia, el $27,8 \%$ también es dependiente ya sea de ambos o de uno de los padres, pero el trabajo es la mayor fuente de sostenimiento (44,6\%). En posgrado cobra mayor fuerza el trabajo, con $57,8 \%$, seguido del padre y esposo .

Tabla 12. Dependencia económica del estudiante, CECAR 2016-1.

\begin{tabular}{|c|c|c|c|c|c|c|c|c|c|c|c|c|c|c|c|c|}
\hline \multirow{3}{*}{ Modalidad } & \multicolumn{16}{|c|}{ Sostenimiento económico } \\
\hline & \multicolumn{2}{|c|}{ Padre } & \multicolumn{2}{|c|}{ Madre } & \multicolumn{2}{|c|}{$\begin{array}{c}\text { Padre y } \\
\text { madre }\end{array}$} & \multicolumn{2}{|c|}{ Otro familiar } & \multicolumn{2}{|c|}{ El trabajo } & \multicolumn{2}{|c|}{ Otro } & \multicolumn{4}{|c|}{ No responde Esposo(a) } \\
\hline & No. & $\%$ & No. & $\%$ & No. & $\%$ & No. & $\%$ & No. & $\%$ & No. & $\%$ & No. & $\%$ & No. & $\%$ \\
\hline PRESENCIAL & 138 & 16,2 & 120 & 14,1 & 340 & 40 & 49 & 5,8 & 165 & 19,4 & 33 & 3,9 & 6 & 0,7 & 0 & 0,0 \\
\hline DISTANCIA & 59 & 7,3 & 50 & 6,2 & 223 & 27,8 & 52 & 6,5 & 358 & 44,6 & 52 & 6,5 & 9 & 1,1 & 0 & 0,0 \\
\hline POSGRADO & 13 & 28,9 & 0 & 0,0 & 0 & 0,0 & 0 & 0,0 & 26 & 57,8 & 0 & 0,0 & 0 & 0,0 & 6 & 13,3 \\
\hline
\end{tabular}

Fuente: cálculo de las autoras con base en la encuesta

Ingresos recibidos. Los datos registrados en la Tabla 13 muestran la oscilación del salario percibido por la mayoría de los estudiantes que trabaja. En posgrado es un poco más del mínimo para el $64,4 \%$, aunque un $22,2 \%$ no percibe ingresos. En pregrado a distancia, el ingreso recibido es de más de un salario mínimo para el $32,8 \%$ y un salario mínimo para el 31,8\%, mientras que no recibe salario el $21 \%$. En presencial, el $47,7 \%$ no percibe salario; entre un salario y un poco más se ubica el $28,6 \%$, el resto no alcanza el salario mínimo. Esta información es de vital importancia al hacer la asignación de becas, trabajo, auxilios, beneficios de almuerzo u otro tipo de ayuda que ofrezca CECAR a los estudiantes.

Tabla 13. Ingresos recibidos por los estudiantes, CECAR 2016-1

\begin{tabular}{|c|c|c|c|c|c|c|c|c|c|c|}
\hline \multirow{3}{*}{ Modalidad } & \multicolumn{10}{|c|}{ Ingresos } \\
\hline & \multicolumn{2}{|c|}{ Un SMLV } & \multicolumn{2}{|c|}{ Medio SMLV } & \multicolumn{2}{|c|}{$\begin{array}{l}\text { Menos de medio } \\
\text { SMLV }\end{array}$} & \multicolumn{2}{|c|}{ Más de un SMLV } & \multicolumn{2}{|c|}{ No recibe salario } \\
\hline & No. & $\%$ & No. & $\%$ & No. & $\%$ & No. & $\%$ & No. & $\%$ \\
\hline PRESENCIAL & 105 & 12,3 & 98 & 11,5 & 103 & 12,1 & 139 & 16,3 & 406 & 47,7 \\
\hline DISTANCIA & 252 & 31,4 & 51 & 6,4 & 68 & 8,5 & 263 & 32,8 & 169 & 21 \\
\hline POSGRADO & 2 & 4,4 & 3 & 6,7 & 1 & 2,2 & 29 & 64,4 & 10 & 22,2 \\
\hline
\end{tabular}

SMLV: salario mínimo legal vigente; Fuente: cálculo de las autoras con base en la encuesta 
Variable gastos educativos. La información que registra la Tabla 14 muestra la forma en que los estudiantes cubren los gastos educativos, y resalta que en los diferentes programas de pregrado la mayoría acude a becas, prestamos, auxilios y ayuda de familiares, mientras que en las especializaciones solo un $38 \%$ recurre a estas formas de pago. El porcentaje de los estudiantes que cubren los gastos educativos con su sueldo asciende al $62 \%$ en posgrado y $35,4 \%$ en la modalidad a distancia. El comportamiento de esta variable se asocia a la condición económica de un número significativo de nuestros estudiantes.

Tabla 14. Formas de cubrimiento de los gastos educativos, CECAR 2016-1.

\begin{tabular}{|c|c|c|c|c|c|c|c|c|c|c|c|c|c|c|c|c|c|c|}
\hline \multirow[t]{2}{*}{ Modalidad } & \multicolumn{2}{|c|}{ Beca } & \multicolumn{2}{|c|}{ Préstamo } & \multicolumn{2}{|c|}{$\begin{array}{l}\text { Sueldo } \\
\text { propio }\end{array}$} & \multicolumn{2}{|c|}{ Auxilio } & \multicolumn{2}{|c|}{ Crédito } & \multicolumn{2}{|c|}{$\begin{array}{c}\text { Ayuda } \\
\text { familiar }\end{array}$} & \multicolumn{2}{|c|}{ Otro } & \multicolumn{2}{|c|}{$\begin{array}{c}\text { No } \\
\text { responde }\end{array}$} & \multicolumn{2}{|c|}{ Empresa } \\
\hline & No. & $\%$ & No. & $\%$ & No. & $\%$ & No. & $\%$ & No. & $\%$ & No. & $\%$ & No. & $\%$ & No. & $\%$ & No. & $\%$ \\
\hline PRESENCIAL & 33 & 3,9 & 234 & 27,5 & 188 & 22,1 & 37 & 4,3 & 264 & 31 & 47 & 5,5 & 39 & 4,6 & 9 & 1,1 & 0 & 0,0 \\
\hline DISTANCIA & 17 & 2,1 & 166 & 20,7 & 284 & 35,4 & 17 & 2,1 & 259 & 32,3 & 22 & 2,7 & 32 & 4 & 6 & 0,7 & 0 & 0,0 \\
\hline POSGRADO & 0 & 0,0 & 4 & 8,9 & 28 & 62 & 4 & 8,9 & 8 & 17,8 & 0 & 0,0 & 0 & 0,0 & 0 & 0,0 & 1 & 2,2 \\
\hline
\end{tabular}

Fuente: cálculo de las autoras con base en la encuesta.

Las variables o características analizadas son comunes a las que establece el Sistema para la Prevención de la Deserción de la Educación Superior - SPADIES para definir el perfil socioeconómico de estudiantes universitarios. Además, guarda cierta similitud con las consideradas en una investigación realizada por la oficina de planeación de la Universidad de Pamplona denominada "Características del perfil socioeconómico del estudiante de pregrado" (2012), que agrupa variables tales como "demográficas", "académicas", "socioeconómicas" y "de bienestar y comportamiento universitario".

\section{CONCLUSIÓN}

En conclusión, en este trabajo de investigación se indagaron las características sociodemográficas y socioeconómicas de los estudiantes de CECAR 2016-I, mediante el uso de instrumentos de la estadística muestral que dan rigor a los resultados obtenidos, entre los cuales se destacan los siguiente:

1. Las condiciones socioeconómicas y familiares de los estudiantes de la Corporación Universitaria del Caribe presentan similitudes entre modalidades y niveles de educación, y resaltan las siguientes características: la mayoría son de estrato uno y dos, lo que se corresponde con el tipo de población que atiende la universidad, la cual, en el marco de su misión, resalta su función social y el compromiso con los grupos más vulnerables del departamento y la región Caribe.

2. En cuanto a la dependencia económica de los padres, esta es mayor en pregrado presencial y a distancia que en posgrado, debido a que a la mayoría de estudiantes se les dificulta estudiar y trabajar a la vez, en el caso de la presencial y los horarios extendidos limitan el vínculo laboral, a lo cual se suman las pocas oportunidades de empleo existentes en la región. 
3. En los ingresos económicos del estudiante y del núcleo familiar, predominan los que no superan los dos salarios mínimos legales, siendo más precarios en pregrado presencial y más altos en posgrado; tales condiciones están asociadas a la escasez de fuentes de empleo a nivel departamental, con predominio de informalidad.

4. Las características socioeconómicas de las familias y del estudiante determinan condiciones como financiamiento de los estudios, egresos o deserción estudiantil, el medio de transporte utilizado, inversión económica en alimentación, materiales de estudio y experiencias laborales temporales.

5. Otra característica que prevalece tanto en estudiantes de pregrado como de postgrado es el estado civil soltero; asimismo, la tendencia de vinculación de estudiante en edades variadas tanto en pregrado presencial como a distancia y posgrado es significativa. Es decir que acuden a las aulas no solo adolescentes y jóvenes sino también personas adultas, aunque con mayor prevalencia en educación a distancia y en las especializaciones.

6. El estudiante de CECAR también se caracteriza por su procedencia de contextos rurales, indígenas, afrodescendientes y de las cabeceras municipales con poco desarrollo socioeconómico. Este factor es considerado positivo; es un indicador de inclusión educativa.

7. El conocimiento profundo y preciso de las características demográficas, biográficas, sociales, familiares y económicas de sus estudiantes y su entorno familiar constituye materia de interés, por una parte, para la gestión académica y fortalecimiento del currículo y, por la otra, para ofrecer un servicio educativo dialogante según las condiciones de vida reales de los estudiantes y su entorno social y familiar.

\section{Recomendaciones}

- Creación e implementación de un sistema de información ágil que capture datos demográficos, económicos, académicos y familiares del estudiante. Por lo que se recomienda realizarlo cada vez que los estudiantes se matriculen en un nuevo semestre; de esta forma se contará con información veraz y oportuna al momento de requerirla.

- Revisión y actualización del "Reglamento de becas y auxilios" en lo relacionado con las opciones y posibilidades de obtención del beneficio, específicamente en los auxilios del Plan Padrino y Auxilios Especiales.

- Gestionar con empresas de la región, proveedores y funcionarios de CECAR el apadrinamiento de estudiantes identificados a través del programa Plan Padrino, previa visita domiciliaria (trabajador social), la matricula financiera, manutención en cuanto a gastos académicos o fotocopias. Con lo anterior, le facilitaría al estudiante en condición de vulnerabilidad su trayectoria académica exitosa al interior de CECAR.

- Implementar desde Bienestar Institucional la creación de la Tienda Universitaria con un capital semilla aprobado por la junta directiva, a través del cual se le oferte a la comunidad universitaria y visitantes materiales de trabajo académico, fotocopia, papelería, souvenir y prendas de vestir, entre otras. Los recursos económicos obtenidos serán destinados al comedor estudiantil impulsado por el Plan Padrino.

- Diseño y creación de una ciudadela o unidad de vivienda universitaria que le permita a los estudiantes de otros municipios y departamentos aledaños contar con el servicio de pensión estudiantil, lo que les facilitaría la estadía y la permanencia en el sistema académico. Se recomienda que los costos de la mensualidad se ajusten al poder adquisitivo de los estudiantes. 


\section{REFERENCIAS}

Acevedo, S. y Jaramillo, A. (2007). Perfil socioeconómico de los estudiantes de pregrado de EAFIT. Medellín: Universidad EAFIT oficina de planeación integral.

Asociación Colombiana de Universidades (ASCUN). (2012). Desarrollo humano sostenible y transformación de la sociedad Política pública para la educación superior y agenda de la Universidad, de cara al país que queremos. Bogotá, Colombia: SAB.

Boucourt, J. y González, M. (2006). Perfil socioeconómico y demográfico del estudiante nuevo ingreso a la Universidad del Zulia. Análisis comparativo. Revista Venezolana de Ciencias Sociales, 10(1).

Corporación Universitaria del Caribe (CECAR). (2015). Plan de Desarrollo Institucional PDI (2016-2020). Sincelejo, Colombia: CECAR.

Corporación Universitaria del Caribe (CECAR). (2015). Proyecto Educativo Institucional - PEI, (2012 - 2016). Sincelejo, Colombia: CECAR.

Departamento Administrativo Nacional de Estadística (DANE). (2012). Encuesta Nacional de Calidad de vida. Colombia.

Díaz, J.; Cañedo, J.; Colindres, S. y Villada, A. (2016). Perfil socioeconómico de mi comunidad. México. Publicado en Educación.

Escuela de Administración, Finanzas y Tecnología (EAFIT). (2007). Estudios socioeconómicos. Recuperado de: http://www.senado.gov.co/funciones.

Gutiérrez de Pineda, V. (1999). Estructura, función y cambio de la familia en Colombia. Medellín: Editorial Universidad de Antioquía.

Hernández, R; Fernández, C. y Baptista, P. (1999). Metodología de la investigación, 6a ed. México: Mc Graw Hill.

Ley 30. Fundamentos de la Educación Superior. Congreso de Colombia. Santafé de Bogotá, Colombia: 28 de diciembre de 1992.

Martínez, M. (2006). Perfil sociodemográfico del turista en el municipio Rangel del Estado de Mérida. En: F. Mártires Ortiz. Diagnóstico del perfil socioeconómico y situación académica de los estudiantes de la Universidad Católica Tecnológica de Barahona UCATEBA (2013). Santo Domingo, República Dominicana: Santillana.

Organización de las Naciones Unidas. (2018). Agenda 2030 y los objetivos de desarrollo sostenible una oportunidad para América Latina y el Caribe. Nueva York: CEPAL. 
Rahona, M. (2006). La influencia del entorno socioeconómico en la realización de estudios universitarios: una aproximación al caso español en la década de los noventa. Hacienda Pública Española, 178(3), 55-80.

Rama, C. (2006). Informe sobre la Educación Superior en América Latina y el Caribe 2000-2005.

Rincón, M. y Semillero de investigación SIGET-CUN. (2016). Perfil socioeconómico y académico de los estudiantes de pregrado del programa de Administración de Empresas de la Corporación Unificada Nacional de Educación Superior (CUN) sede Bogotá, centro 2015. Revista UNIMAR, 34(1), 131-145.

UNESCO. (2006). Informe sobre la educación superior en América Latina y el Caribe 2000-2005. Caracas, Venezuela: Metrópolis.

Universidad Autónoma de Occidente. (2007). Perfil socioeconómico del estudiante autónomo. Cali, Colombia: Universidad Autónoma de Occidente.

Universidad de Pamplona. (2012). Características del perfil socioeconómico del estudiante de pregrado de la universidad de pamplona. Pamplona, Colombia: Oficina de planeación.

Universidad Nacional de Colombia. (2001). Perfil socioeconómico de los estudiantes de la universidad nacional. Bogotá: Universidad Nacional de Colombia.

Willianson. J. (1991). Diagnóstico del perfil socioeconómico y situación académica de los estudiantes de la Universidad Católica Tecnológica de Barahona UCATEBA (2013). [Trabajo de investigación]. F. Ortiz (coord.). Santo Domingo, República Dominicana. 